\title{
Homotopy nilpotent groups
}

\author{
GEORG BIEDERMANN \\ WILLIAM G DWYER
}

\begin{abstract}
We study the connection between the Goodwillie tower of the identity and the lower central series of the loop group on connected spaces. We define homotopy $n$-nilpotent groups as homotopy algebras over certain simplicial algebraic theories. This notion interpolates between infinite loop spaces and loop spaces, but backwards. We study the relation to ordinary nilpotent groups. We prove that $n$-excisive functors of the form $\Omega F$ factor over the category of homotopy $n$-nilpotent groups.
\end{abstract}

55P47, 55U35; 18C10, 55P35

\section{Introduction}

This article arose from the question of what the Goodwillie tower of the identity has to do with the lower central series of Kan's loop group of a connected space. We express the relation with the help of simplicial algebraic theories. We expect the reader to be familiar with the basic notions of homotopical algebra and Goodwillie's calculus of homotopy functors. We will give a short introduction to simplicial algebraic theories and their homotopy algebras in Sections 2 and 3.

We are working simplicially, so for us "space" always means "simplicial set".

Let us introduce some notation valid for the rest of the article. Let $\mathcal{S}_{*}$ be the category of pointed simplicial sets. Further let $\mathcal{F}$ denote the category of $\mathcal{S}_{*}$-functors from finite pointed simplicial sets $\mathcal{S}_{*}^{\text {fin }}$ to $\mathcal{S}_{*}$. A homotopy functor in $\mathcal{F}$ is a functor that preserves weak equivalences.

For a homotopy functor $F$ in $\mathcal{F}$ Goodwillie [20] constructs a tower of functors

$$
F \rightarrow \cdots \rightarrow P_{n} F \rightarrow P_{n-1} F \rightarrow \cdots \rightarrow P_{1} F \rightarrow P_{0} F=F(*),
$$

where the $n$-th stage is the universal $n$-excisive homotopy functor under $F$. Here, $n$-excision is a higher version of excision; a 1-excisive functor is a homotopy functor with a Mayer-Vietoris sequence. For $F=$ id, the Goodwillie tower converges on simply connected spaces to the identity; see Goodwillie [19]. On a connected space $K$, 
however, it converges to the Bousfield-Kan completion proved in [3] by Arone and Kankaanrinta:

$$
\underset{n}{\operatorname{holim}} P_{n}(\mathrm{id})(K) \simeq \mathbb{Z}_{\infty} K
$$

We have $P_{1}$ (id) $\simeq \Omega^{\infty} \Sigma^{\infty}$, the stable homotopy functor. So the Goodwillie tower interpolates between stable and unstable homotopy. The map $K \rightarrow P_{n}(\mathrm{id})(K)$ is roughly $(n+1) k$-connected, if $K$ is $k$-connected.

Let $\mathcal{S}_{0}$ be the category of reduced simplicial sets, ie simplicial sets with exactly one $0-$ simplex. By a result of Dwyer and Kan [15], Kan's loop group functor $G$ is part of a Quillen equivalence between $\mathcal{S}_{0}$ and simplicial groups. The homotopy category of $\mathcal{S}_{0}$ and of path-connected topological spaces are equivalent.

For a reduced simplicial set $K$, let $G K / \Gamma_{n} G K$ denote the $n$-th stage of the lower central series of $G K$ taken degreewise as defined in Definition 6.1. This was studied by Curtis [12] who proved that for simply connected spaces the connectivity of the map

$$
G K \rightarrow G K / \Gamma_{n+1} G K
$$

increases logarithmically with $n$. The tower $\left\{G K / \Gamma_{n+1} G K\right\}_{n \geq 1}$ associated to the filtration converges to the identity on simply connected spaces. As noted by Kan,

$$
\pi_{s-1}\left(G K / \Gamma_{2} G K\right) \cong H_{S} K \text { for all } s \geq 1 .
$$

So the lower central series interpolates between singular homology and unstable homotopy. In a similar way to the Goodwillie tower of id, this tower converges to the identity on simply connected spaces and to the Bousfield-Kan completion on connected spaces.

It is not difficult to check that there is a canonical map up to homotopy

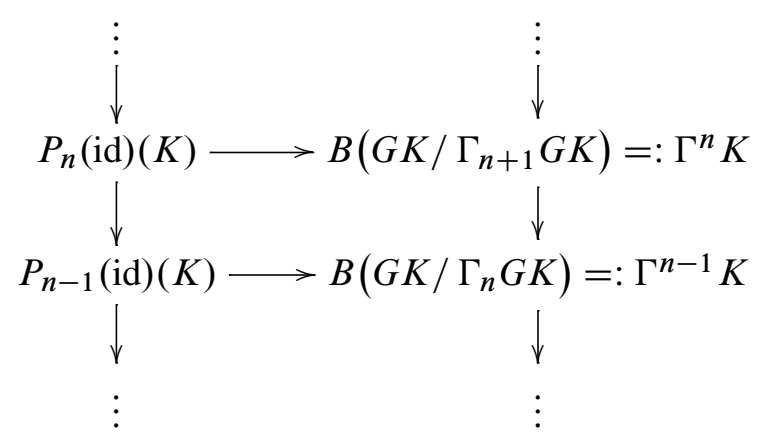

from the Goodwillie tower of the identity of $K$ to the classifying space of the lower central series of the loop group of $K$, obtained from the universal property (up to homotopy) of the first tower. It is well-known to the experts that this map induces an isomorphism on $\pi_{1}$. We prove this statement in Theorem 8.1 and phrase it in terms 
of algebraic theories. In fact, the left hand side (looped once) gives rise to certain simplicial algebraic theories that we call $\mathcal{G}_{n}$ (Definition 5.2). Applying $\pi_{0}$ to this simplicial category gives us the ordinary theory of $n$-nilpotent groups. The $k$-ary operations of these theories can be obtained by looping the above towers and evaluating them on a $k$-fold wedge of circles (Corollary 5.7).

Algebraic theories were introduced by Lawvere [27] to obtain categorical descriptions of algebraic structures like groups, rings, Lie algebras, etc. An algebraic theory is a category $T$ having the natural numbers $k \geq 0$ as objects such that $k$ is the product in $T$ of $k$ copies of 1 . The maps from $k$ to 1 are to be thought of as the $k$-ary operations of $T$. They can be canonically identified with the free objects on $k$ generators. Algebras over $T$ are product preserving functors from $T$ to sets.

For purposes in homotopy theory we need to consider simplicial algebraic theories where $T$-algebras have values in simplicial sets. These were first considered by Reedy [30] and more recently by Schwede [31] and Badzioch [5]. It is convenient for us to study pointed versions where algebras are functors from $T$ to pointed simplicial sets and the category $T$ itself will be enriched over $\mathcal{S}_{*}$. If the theory has only one constant - as in our case - there is no loss in generality. We also need a weaker notion of algebra: homotopy $T$-algebras were introduced by Badzioch [5]. They are functors from $T$ to $\mathcal{S}_{*}$ that commute with products up to homotopy.

We define a homotopy $n$-nilpotent group to be a homotopy $\mathcal{G}_{n}$-algebra. In other words, these are spaces that admit $k$-ary operations parametrized by $\Omega P_{n}(\mathrm{id})\left(\bigvee_{k} S^{1}\right)$. Homotopy $n$-nilpotent groups have not been considered before - only the two extreme cases are classical: Loop spaces and infinite loop spaces can be described as homotopy algebras over our theories $\mathcal{G}_{\infty}$ and $\mathcal{G}_{1}$; see Theorem 5.8 and Theorem 5.13. The free objects on $k$ generators of these simplicial theories are given by

$$
\Omega\left(\bigvee_{i=1}^{k} S^{1}\right) \simeq \Omega \Sigma \bigvee_{k} S^{0} \text { and } \Omega P_{1}(\mathrm{id})\left(\bigvee_{i=1}^{k} S^{1}\right) \simeq \Omega^{\infty} \Sigma^{\infty} \bigvee_{k} S^{0} .
$$

Thus, the new notion interpolates between loop spaces and infinite loop space, but "backwards".

We obtain morphisms of theories

$$
\mathcal{G}_{\infty} \rightarrow \cdots \rightarrow \mathcal{G}_{n} \rightarrow \mathcal{G}_{n-1} \rightarrow \cdots
$$

induced by the maps in the Goodwillie tower and functors

$$
U_{n}: \mathcal{S}_{*}^{\mathcal{G}_{n}} \rightarrow \mathcal{S}_{*}^{\mathcal{G}_{\infty}} .
$$


The functor $U_{n}$ induces on homotopy categories of the respective Badzioch model structures the forgetful functor from homotopy $n$-nilpotent groups to loop spaces. It has a left adjoint

$$
\Phi_{n}: \mathcal{S}_{*}^{\mathcal{G}} \rightarrow \mathcal{S}_{*}^{\mathcal{G}_{n}}
$$

It is easy to check that $\left(\Phi_{n}, U_{n}\right)$ form a Quillen pair for the Badzioch model structures on each side, and hence their derived functors $\left(L \Phi_{n}, R U_{n}\right)$ form an adjoint pair of functors on the respective homotopy categories. Left adjoints to forgetful functors are often called free functors. For a connected space $X$ we have $\Omega X \simeq \underset{\sim}{X}\left(1^{+}\right)$where $\underset{\sim}{X}: \mathcal{G}_{\infty} \rightarrow \mathcal{S}_{*}$ is a homotopy $\infty$-algebra. In that sense the object $L \Phi_{n}(\underset{\sim}{X})$ is the free homotopy $n$-nilpotent group generated by $\Omega X$. Our next result, Theorem 5.16 , exhibits the Goodwillie tower of the identity $\Omega P_{n}(\mathrm{id})(X)$ as the homotopy left Kan extension along $\Phi_{n}$ :

$$
\Omega P_{n}(\mathrm{id})(X) \simeq\left(L \Phi_{n} \underset{\sim}{X}\right)\left(1^{+}\right) .
$$

So the free homotopy $n$-nilpotent group generated by $\Omega X$ is $\Omega P_{n}(\mathrm{id})(X)$. Equivalently, Theorem 5.16 states that the free homotopy $n$-nilpotent group generated by a pointed space $K$ is given by $\Omega P_{n}(\mathrm{id})(\Sigma K)$. This generalizes and interpolates between the fact that $\Omega \Sigma K$ is the free loop space and $\Omega^{\infty} \Sigma^{\infty} K$ is the free infinite loop space over $K$. If the reader prefers the analogy with the lower central series, then the case $n=1$ corresponds to the abelianization of a group and $\Omega^{\infty} \Sigma^{\infty} K$ is the homotopy 1 -nilpotent quotient of $\Omega \Sigma K$. Then for $n \geq 1$ the term $\Omega P_{n}(\mathrm{id})(\Sigma K)$ can be seen as a homotopy version of the quotients in the lower central series.

Corollary 9.3 gives a large source of examples: a space is a homotopy $n$-nilpotent group if it is the value of a looped $n$-excisive functor. In work in progress [8] we prove that every homotopy $n$-nilpotent group arises in this way. An application, Corollary 9.4 , is that the category of looped $n$-excisive functors is naturally enriched in homotopy $n$-nilpotent groups. This generalizes the fact that the homotopy category of infinite loop spaces is enriched over abelian groups.

In Example 9.8 we give an easy example of homotopy $n$-nilpotent group: every connected space whose homotopy groups vanish except between dimensions $k+1$ and $(n+1) k$ for some $k \geq 1$ is canonically a homotopy $n$-nilpotent group. We finish with an application, Example 9.10, of the chain rule on Goodwillie's calculus of homotopy functors proved by Arone and Ching in [1] that states that homotopy functors, whose derivatives vanish outside a certain range, factor over the category of homotopy $n$-nilpotent spaces. This generalizes and simplifies the proof of the corresponding statement for $n=1$ by Arone, Dwyer and Lesh in [2]. 
The results in this article are not necessarily deep. This article is meant to formulate the definitions and set the ground for further study of homotopy $n$-nilpotent spaces. We hope to convince the reader that this subject deserves further attention.

Acknowledgments We would like to thank André Joyal and Gerald Gaudens for several stimulating discussions and Bernard Badzioch for explaining Theorem 3.5 to us. We would also like to thank the referee. The research for this paper was started while both authors were guests at the Thematic Program on Geometric Applications of Homotopy Theory at the Fields Institute for Mathematics, Toronto. The first author was a postdoctoral fellow at the University of Western Ontario, London, Ontario. The paper was finished while the first author was a guest at the Max-Planck-Institut für Mathematik in Bonn and Assistant Professor at the Universidad de los Andes, Bogotá, Colombia.

\section{Algebraic theories}

Algebraic theories were introduced by Lawvere [27] in order to describe algebraic structures, like eg groups or rings, in terms of universal operations. He found that these universal operations are encoded by the homomorphisms between the finitely generated free objects of the respective algebraic structure. Thus the typical example of an algebraic theory is given by the subcategory of some algebraic category formed by the finitely generated free objects. Any object of the algebraic category in question determines and is determined by a functor from this subcategory to sets. Since we prefer to deal with covariant functors we consider the opposite of the subcategory of finitely generated free objects, and since we will deal only with pointed objects we describe here only pointed algebraic theories.

Definition 2.1 For a category $\mathcal{C}$ we will refer to its set of morphisms by $\mathcal{C}\left({ }_{-},{ }_{-}\right)$.

Definition 2.2 Let $\Gamma$ be the opposite of the category of finite pointed sets. The category $\Gamma$ has all products and every object is isomorphic to an object of the form

$$
k^{+}=\{1, \ldots, k\} \cup\{+\} .
$$

Here + acts as the base point. For every $1 \leq s \leq k$ we have maps $i_{s}^{k}: k^{+} \rightarrow 1^{+}$given by the inclusion of the pointed set $1^{+}$to $k^{+}$where the non basepoint of $1^{+}$maps to $s \in k^{+}$. These maps induce an isomorphism

$$
\prod_{s=1}^{k} i_{s}^{k}: k^{+} \cong \prod_{s=1}^{k} 1^{+} .
$$


Definition 2.3 A pointed algebraic theory is a category $T$ having the same set of objects as $\Gamma$ together with a functor $\Gamma \rightarrow T$, which is the identity on objects and preserves products. Morphisms of algebraic theories are product preserving functors under $\Gamma$.

The category $T$ is usually given as a full subcategory of some other category (see Remark 2.9), so the morphisms in $T$ are often left understood. We will often confuse the objects $k^{+} \in \mathrm{Ob}(\Gamma)=\mathrm{Ob}(T)$ with their images under such a full inclusion. If we want to emphasize a particular theory $T$, we will denote the object $k^{+}$by $T\left(k^{+}\right)$.

Definition 2.4 A strict $T$-algebra is a functor $\underset{\sim}{X}: T \rightarrow$ Sets that preserves products strictly. This means that the map

$$
\prod_{s=1}^{k} \underset{\sim}{X}\left(i_{s}^{k}\right): \underset{\sim}{X}\left(k^{+}\right) \rightarrow\left(\underset{\sim}{X}\left(1^{+}\right)\right)^{k}
$$

is an isomorphism of pointed sets. The category of pointed $T$-algebras is denoted by $\mathrm{Alg}_{T, *}$ and forms a full subcategory of the category $\operatorname{Sets}_{*}^{T}$ of all functors from $T$ to pointed sets.

Definition 2.5 The $k$-ary operations of an algebraic theory $T$ are the elements of the set $T\left(k^{+}, 1^{+}\right)$. The constants of $T$ are the 0 -ary operations, ie the elements of the set

$$
A_{0}=T\left(0^{+}, 1^{+}\right) \text {. }
$$

A theory with one constant is an algebraic theory such that $A_{0} \cong *$.

Remark 2.6 Let $T$ be an algebraic theory. Then the forgetful functor $u$ : Sets $* \rightarrow$ Sets induces a functor

$$
u_{*}: \operatorname{Sets}_{*}^{T} \rightarrow \operatorname{Sets}^{T}
$$

This restricts to a functor

$$
u_{*}: \operatorname{Alg}_{T, *} \rightarrow \operatorname{Alg}_{T},
$$

where $\operatorname{Alg}_{T}$ is the category of unpointed $T$-algebras.

Lemma 2.7 If $T$ is an algebraic theory with one constant, the functor

$$
u_{*}: \operatorname{Alg}_{T, *} \rightarrow \operatorname{Alg}_{T}
$$

is an isomorphism of categories. 
Proof Given an unpointed $T$-algebra $X$, we can always supply it with a canonical basepoint

$$
* \cong X\left(0^{+}\right) \rightarrow X\left(1^{+}\right),
$$

induced by the unique constant $0^{+} \rightarrow 1^{+}$in $T$. We obtain an inverse functor for $u_{*}$. $\square$

Definition 2.8 The evaluation functor

$$
\operatorname{Ev}_{1+}: \operatorname{Alg}_{*}^{T} \rightarrow \operatorname{Sets}_{*}, \underset{\sim}{X} \mapsto \operatorname{Ev}_{1}+\underset{\sim}{X}=\underset{\sim}{X}\left(1^{+}\right)
$$

has a left adjoint denoted by

$$
\mathrm{Fr}_{T}: \mathrm{Sets}_{*} \rightarrow \operatorname{Alg}_{T, *}
$$

which is called the free $T$-algebra functor.

Remark 2.9 One easily checks that in $\operatorname{Alg}_{T, *}$ there is a canonical isomorphism

$$
\operatorname{Fr}_{T}\left(k^{+}\right) \cong T\left(k^{+},{ }_{-}\right) \text {. }
$$

Hence there is an equivalence of categories between $T$ and the opposite category of the full subcategory of $\mathrm{Alg}_{T, *}$ given by the finitely generated free $T$-algebras.

Remark 2.10 To illustrate the ideas we describe here very briefly the example of the algebraic theory of groups. Let $\mathrm{Gr}$ be the category of groups. We let $\mathrm{Nil}_{\infty}$ be the theory of groups.

According to Remark 2.9 the object $k^{+}$of the theory Nil $\infty_{\infty}$ of groups can be identified with

$$
\operatorname{Nil}_{\infty}\left(k^{+}\right)=F_{k},
$$

the free group on $k$ letters. Morphisms are given by the set

$$
\operatorname{Nil}_{\infty}\left(k^{+}, \ell^{+}\right) \cong \operatorname{Gr}\left(F_{\ell}, F_{k}\right) \cong\left(F_{k}\right)^{\ell} .
$$

One easily checks that composition

$$
\operatorname{Nil}_{\infty}\left(\ell^{+}, m^{+}\right) \times \operatorname{Nil}_{\infty}\left(k^{+}, \ell^{+}\right) \rightarrow \operatorname{Nil}_{\infty}\left(k^{+}, m^{+}\right)
$$

translates to substitution $\left(F_{\ell}\right)^{m} \times\left(F_{k}\right)^{\ell} \rightarrow\left(F_{k}\right)^{m}$, where we take $\ell$ words $w_{1}, \ldots, w_{\ell}$ in $F_{k}$ and substitute them for the generators of $F_{\ell}$.

Now suppose that $G$ is a group. We can define a functor $\underset{\sim}{G}: \mathrm{Nil}_{\infty} \rightarrow$ Sets by setting

$$
k^{+} \mapsto G^{k}
$$


and for every element in $\left(w_{1}, \ldots, w_{k}\right) \in\left(F_{k}\right)^{\ell} \cong \mathrm{Nil}_{\infty}\left(k^{+}, \ell^{+}\right)$we define a group homomorphism $G^{k} \rightarrow G^{\ell}$ given by

$$
\left(g_{1}, \ldots, g_{k}\right) \mapsto\left(h_{1}, \ldots, h_{\ell}\right),
$$

where $h_{j}$ for $1 \leq j \leq \ell$ is the element in $G$ obtained by substituting $g_{i}$ for the $i$-th generator of $F_{k}$ in the word $w_{i}$ for $1 \leq i \leq k$. It is straightforward to check that this is indeed a product-preserving functor, hence a $\mathrm{Nil}_{\infty}$-algebra.

Conversely, given an $\mathrm{Nil}_{\infty}$-algebra $\underset{\sim}{G}$ we obtain a group structure on the set $\underset{\sim}{G}\left(1^{+}\right)=$: $G$. A multiplication map

$$
G \times G \cong \underset{\sim}{G}\left(2^{+}\right) \rightarrow \underset{\sim}{G}\left(1^{+}\right) \cong G
$$

is induced by the element

$$
f_{1} f_{2} \in F_{2} \cong \operatorname{Nil}_{\infty}\left(2^{+}, 1^{+}\right) .
$$

Here we denote by $f_{1}, \ldots, f_{k}$ the generators of the free group $F_{k}$. This multiplication is associative because of the identity

$$
\left(f_{1} f_{2}\right) f_{3}=f_{1}\left(f_{2} f_{3}\right) \in F_{3} \cong \operatorname{Nil}_{\infty}\left(3^{+}, 1^{+}\right) .
$$

The set $G$ is pointed and one easily checks that this basepoint $e$ is the neutral element with respect to the multiplication. The inverse $\left({ }_{-}\right)^{-1}: G \rightarrow G$ is induced by the element $f_{1}^{-1} \in F_{1}$. Substituting $f_{1}^{-1}$ for $f_{2}$ we obtain the identity $f_{1} f_{1}^{-1}=e$ which proves the inverse as defined is indeed an inverse.

Both construction are mutually inverse and give an equivalence between the category $\mathrm{Gr}$ of groups and the category of $\mathrm{Nil}_{\infty}$-algebras. In turn, this illustrates the isomorphism of the opposite of the full subcategory of Gr given by the finitely generated free groups and the theory $\mathrm{Nil}_{\infty}$.

An example of an ordinary algebraic theory that has more than one constant, is the theory of commutative rings with unit. The free object on $k$ generators is given by $\mathbb{Z}\left[x_{1}, \ldots, x_{k}\right]$, the polynomial ring in $k$ generators. The constants are given by $A_{0} \cong \mathbb{Z}$.

\section{Simplicial algebraic theories}

We denote the category of pointed simplicial sets by $\mathcal{S}_{*}$.

Definition 3.1 A pointed simplicial algebraic theory is an algebraic theory enriched as a category over $\mathcal{S}_{*}$. We will immediately drop all adjectives and simply speak of a theory henceforth. 
Definition 3.2 Let $T$ be a theory. A pointed strict simplicial $T$-algebra is an $\mathcal{S}_{*}-$ functor from $T$ to $\mathcal{S}_{*}$ that preserves products in the sense of Definition 2.4. Again, we will simply speak of a $T$-algebra from now on. The category of $T$-algebras will be denoted by $\operatorname{Alg}_{T, *}$.

Since we want to do homotopy theory we relax the conditions on $T$-algebras.

Definition 3.3 Let $T$ be a theory. A homotopy T-algebra $\underset{\sim}{X}$ is an $\mathcal{S}_{*}$-functor from $T$ to $\mathcal{S}_{*}$ that preserves products up to weak equivalence, ie the map

$$
\prod_{s=1}^{k} \underset{\sim}{X}\left(i_{s}^{k}\right): \underset{\sim}{X}\left(k^{+}\right) \rightarrow\left(\underset{\sim}{X}\left(1^{+}\right)\right)^{k}
$$

is a weak equivalence for all $k \geq 1$.

The category $\mathcal{S}_{*}^{T}$ can be equipped with a model structure where the objectwise fibrant homotopy $T$-algebras are exactly the fibrant objects. This model structure was first constructed by Badzioch in [5] and is a localization of the projective model structure on $\mathcal{S}_{*}^{T}$.

Definition 3.4 Badzioch in [5] called this model structure the homotopy algebra model structure. We will call it the Badzioch model structure and denote the category $\mathcal{S}_{*}^{T}$ together with this model structure by $\left(\mathcal{S}_{*}^{T}\right)_{\mathrm{Badz}}$.

It is shown in [5] that there is a Quillen equivalence between strict $T$-algebras with objectwise weak equivalences and fibrations and homotopy $T$-algebras. This result tells us that - independently of the theory $T$ - any homotopy $T$-algebra can be rigidified.

Theorem 3.5 (Badzioch) Let $F: S \rightarrow T$ be a morphism of simplicial theories. If $F$ is a weak equivalence of simplicial categories then pulling back along $F$ is the right adjoint of a Quillen equivalence between the associated Badzioch model categories.

Proof In [16, Theorem 2.1] it is shown that $F^{*}: \mathcal{S}_{*}^{T} \rightarrow \mathcal{S}_{*}^{S}$ is the right adjoint of a Quillen equivalence between the projective model structures. The Badzioch model structures are left Bousfield localizations. This process preserves Quillen equivalences by [22, Theorem 3.3.20].

Remark 3.6 As in the set-valued case the evaluation functor

$$
\mathrm{Ev}_{1^{+}}: \operatorname{Alg}_{T, *} \rightarrow \mathcal{S}_{*}, \underset{\sim}{X} \mapsto \mathrm{Ev}_{1}+\underset{\sim}{X}=\underset{\sim}{X}\left(1^{+}\right)
$$


has an $\mathcal{S}_{*}$-enriched left adjoint denoted by

$$
\operatorname{Fr}_{T}: \mathcal{S}_{*} \rightarrow \operatorname{Alg}_{T, *}
$$

which is called the free $T$-algebra functor. One easily checks that $\left(\operatorname{Fr}_{T}, \operatorname{Ev}_{T}\right)$ form a Quillen pair for the Badzioch model structure and that there is a canonical isomorphism

$$
\operatorname{Fr}_{T}\left(k^{+}\right) \cong T\left(k^{+},{ }_{-}\right) .
$$

\section{The $n$-excisive model structure}

Definition 4.1 We denote by $\mathcal{F}$ the category of $\mathcal{S}_{*}$-enriched functors from finite pointed simplicial sets $\mathcal{S}_{*}^{\text {fin }}$ to pointed simplicial sets $\mathcal{S}_{*}$.

The category $\mathcal{F}$ is enriched, tensored and cotensored over $\mathcal{S}_{*}$ where both tensor and cotensor are given objectwise. It carries a projective model structure where weak equivalences and fibrations are given objectwise.

For an introduction to Goodwillie's calculus of homotopy functors and in particular for the notion of $n$-excisive homotopy functor we refer to Goodwillie [20] and Kuhn [26].

In Biedermann, Chorny and Röndigs [7] and Dwyer [14] the projective model structure on $\mathcal{F}$ was localized to obtain the $n$-excisive model structure where the fibrant objects are exactly the $n$-excisive homotopy functors. A map $\underset{\sim}{X} \rightarrow \underset{\sim}{Y}$ is an $n$-excisive weak equivalence if and only if it induces an objectwise weak equivalence

$$
P_{n} \underset{\sim}{X} \rightarrow P_{n} \underset{\sim}{Y} .
$$

Here $P_{n} \underset{\sim}{X}$ denotes the $n$-th stage in the Goodwillie tower of the functor $\underset{\sim}{X}{ }^{h}$ which is the functor $\underset{\sim}{X}$ pre- and postcomposed with an objectwise fibrant replacement functor in $\mathcal{S}_{*}$. However it is more convenient for us to consider the injective model structure on $\mathcal{F}$ constructed by Joyal [25] and Jardine [23] where cofibrations are given by all inclusions. This model structure is also proper and simplicial with the advantage that all objects are cofibrant. The same techniques as in [7] apply to arrive at an $n$-excisive model structure on $\mathcal{F}$ with the same equivalences and where a map $\underset{\sim}{X} \rightarrow \underset{\sim}{Y}$ is an $n$-excisive fibration if and only if it is an injective fibration such that the square

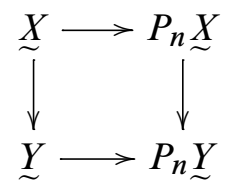

is an objectwise homotopy pullback square. We have: 
Theorem 4.2 The injective $n$-excisive model structure on $\mathcal{F}$ is a cofibrantly generated proper simplicial model structure. All objects are cofibrant.

Corollary 4.3 Let $\underset{\sim}{Y}$ be an injectively fibrant $n$-excisive homotopy functor. Then for every $\underset{\sim}{X}$ in $\mathcal{F}$ we have a natural weak equivalence

$$
\mathcal{F}\left(P_{n} \underset{\sim}{X} \underset{\sim}{Y}\right) \simeq \mathcal{F}(\underset{\sim}{X}, \underset{\sim}{Y})
$$

\section{Homotopy $n$-nilpotent groups}

We will now describe the simplicial theory of homotopy $n$-nilpotent groups.

Definition 5.1 In the category $\mathcal{F}$ let $\left(_{-}\right)^{\text {inj }}$ be a fibrant replacement functor with respect to the injective model structure.

Definition 5.2 We define a full subcategory $\mathcal{G}_{n}$ of the category $\mathcal{F}$, which has for each natural number $k \geq 0$ exactly one object given by

$$
\mathcal{G}_{n}\left(k^{+}\right)=\prod_{i=1}^{k} \Omega\left(P_{n}(\mathrm{id})\right)^{\mathrm{inj}} .
$$

We also define for $n=\infty$ the category $\mathcal{G}_{\infty}$ with objects given by

$$
\mathcal{G}_{\infty}\left(k^{+}\right)=\prod_{i=1}^{k} \Omega(\mathrm{id})^{\mathrm{inj}} .
$$

We employ the convention that the empty product is the final object $*$, and so we have for all $1 \leq n \leq \infty$ and $0 \leq k<\infty$ :

$$
\mathcal{G}_{n}\left(k^{+}\right) \cong \prod_{i=1}^{k} \mathcal{G}_{n}\left(1^{+}\right) .
$$

We let $I_{n}: \mathcal{G}_{n} \rightarrow \mathcal{F}$ be the inclusion functor.

To obtain the status of a theory, $\mathcal{G}_{n}$ has to live under $\Gamma$. So we define a functor $\gamma_{n}: \Gamma \rightarrow \mathcal{G}_{n}$ on objects simply by $k^{+} \mapsto \mathcal{G}_{n}\left(k^{+}\right)$. The space $\mathcal{G}_{n}\left(k^{+}\right)$is the space of pointed maps from $k^{+}$to $\mathcal{G}_{n}\left(1^{+}\right)$. A morphism $f^{\text {op }:} \ell^{+} \rightarrow k^{+}$in $\Gamma$, which is represented by a map $f: k^{+} \rightarrow \ell^{+}$of pointed sets, then induces a map

$$
\mathcal{G}_{n}\left(\ell^{+}\right) \rightarrow \mathcal{G}_{n}\left(k^{+}\right)
$$

by pulling back along $f$. 
Remark 5.3 As a full subcategory of $\mathcal{F}$ the category $\mathcal{G}_{n}$ for $1 \leq n \leq \infty$ is enriched over $\mathcal{S}_{*}$. The category $\mathcal{G}_{n}$ constitutes a simplicial theory as discussed in Section 3 . Therefore we can consider $\mathcal{G}_{n}$-algebras and homotopy $\mathcal{G}_{n}$-algebras. Objectwise fibrant homotopy $\mathcal{G}_{n}$-algebras are the fibrant objects in $\left(\mathcal{S}_{*}^{\mathcal{G}_{n}}\right)_{\text {Badz }}$.

Definition 5.4 We call a homotopy $\mathcal{G}_{n}$-algebra a homotopy $n$-nilpotent group. If the context allows, we commit the common abuse of language and refer to the underlying space $\underset{\sim}{X}\left(1^{+}\right)$(or one canonically weakly equivalent to it) for some homotopy $\mathcal{G}_{n^{-}}$ algebra $\underset{\sim}{X}$ as a homotopy $n$-nilpotent group.

For $n=1$ this corresponds to the common abuse of language to refer to an infinite loop space by its underlying space without making the infinite loop space structure explicit. Obviously, a space might not have any infinite loop space structure, and if it has one it might have more than one. Similarly for homotopy $n$-nilpotent groups.

Lemma 5.5 For all $n \geq 1$ and $k, \ell \geq 0$ we have canonical weak equivalences

$$
\mathcal{G}_{n}\left(k^{+}, \ell^{+}\right) \simeq \prod_{\ell} \Omega P_{n}(\mathrm{id})\left(\bigvee_{i=1}^{k} S^{1}\right)
$$

Proof Recall that $\mathcal{F}$ from Definition 4.1 is endowed with the injective $n$-excisive model structure where all objects are cofibrant. We compute:

$$
\begin{aligned}
\mathcal{G}_{n}\left(k^{+}, \ell^{+}\right) & \cong \mathcal{F}\left(\prod_{k} \Omega\left(P_{n}(\mathrm{id})\right)^{\mathrm{inj}}, \prod_{\ell} \Omega\left(P_{n}(\mathrm{id})\right)^{\mathrm{inj}}\right) \\
& \simeq \mathcal{F}\left(\Omega(\mathrm{id})^{k}, \Omega\left(P_{n}(\mathrm{id})\right)^{\mathrm{inj}}\right)^{\ell} \\
& \cong \mathcal{F}\left(\operatorname{map}_{\mathcal{S}_{*}}\left(\bigvee_{k} S^{1},{ }_{-}\right), \Omega\left(P_{n}(\mathrm{id})\right)^{\mathrm{inj}}\right)^{\ell} \\
& \cong\left(\Omega\left(P_{n}(\mathrm{id})\right)^{\mathrm{inj}}\left(\bigvee_{k} S^{1}\right)\right)^{\ell} \\
& \simeq\left(\Omega P_{n}(\mathrm{id})\left(\bigvee_{k} S^{1}\right)\right)^{\ell}
\end{aligned}
$$

The weak equivalence in step 2 comes from Corollary 4.3. We also use the enriched Yoneda lemma and the fact that $P_{n}$ commutes with $\Omega$.

Remark 5.6 By Lemma 5.5 the theory $\mathcal{G}_{n}$ has only one constant. So by Lemma 2.7 we can work in the pointed setting without losing information. 
Corollary 5.7 The $k$-ary operations of the theory $\mathcal{G}_{n}$ of homotopy $n$-nilpotent groups is given by evaluating the free $\mathcal{G}_{n}$-algebra on $k$ generators at $1^{+}$:

$$
\operatorname{Ev}_{1}+\left(\operatorname{Fr}_{\mathcal{G}_{n}}\left(k^{+}\right)\right) \cong \Omega P_{n}(\mathrm{id})\left(\bigvee_{i=1}^{k} S^{1}\right)
$$

Proof The free algebra $A_{k}$ on $k$ generators in any simplicial theory $T$ can be obtained by the following formula:

$A_{k} \cong T\left(k^{+}, 1^{+}\right) \cong \operatorname{Alg}_{T, *}\left(\operatorname{Fr}_{T}\left(1^{+}\right), \operatorname{Fr}_{T}\left(k^{+}\right)\right) \cong \operatorname{Fr}_{T}\left(k^{+}\right)\left(1^{+}\right) \cong \operatorname{Ev}_{1^{+}}\left(\operatorname{Fr}_{T}\left(k^{+}\right)\right)$.

Now the statement follows from Lemma 5.5.

If $F_{k}$ is the free group on $k$ generators then we have the following canonical weak equivalences:

$$
\mathcal{G}_{\infty}\left(k^{+}, 1^{+}\right) \simeq \Omega \bigvee_{k} S^{1} \simeq \Omega B F_{k} \simeq F_{k}
$$

It follows that the theory $\mathcal{G}_{\infty}$ is weakly equivalent as a simplicial category to the discrete theory of groups.

Theorem 5.8 The category $\left(\mathcal{S}_{*}^{\mathcal{G}}{ }^{\infty}\right)_{\text {Badz }}$ is Quillen equivalent to the category of simplicial groups. In particular, the homotopy category of homotopy $\infty$-nilpotent groups is equivalent to the homotopy category of loop spaces.

Proof This follows readily from the equivalences (1) and Theorem 3.5.

In [6] Badzioch, Chung and Voronov define a theory $\mathcal{T}_{n}$ such that its homotopy algebras are exactly $n$-fold loop spaces.

Definition 5.9 Set $\mathcal{T}_{n}\left(k^{+}\right)=\bigvee_{k} S^{n}$ and take as morphisms the derived mapping space

$$
\mathcal{T}_{n}\left(k^{+}, \ell^{+}\right)=\operatorname{map}_{\mathcal{S}_{*}}^{\operatorname{der}}\left(\bigvee_{\ell} S^{n}, \bigvee_{k} S^{n}\right)
$$

So $\mathcal{T}_{n}$ is the opposite of the full subcategory of $\mathcal{S}_{*}$ given by the finite wedges of $n$-spheres. The suspension functor induces a morphism $t_{n}: \mathcal{T}_{n} \rightarrow \mathcal{T}_{n+1}$ of simplicial theories:

$$
\mathcal{T}_{n}\left(k^{+}, \ell^{+}\right)=\left(\Omega^{n} \bigvee_{k} S^{n}\right)^{\ell} \rightarrow\left(\Omega^{n+1} \bigvee_{k} S^{n+1}\right)^{\ell} \cong \mathcal{T}_{n+1}\left(k^{+}, \ell^{+}\right)
$$


Theorem 5.10 [6, Theorem 1.1] A pointed space $X$ is an $n$-fold loop space if and only if there exists a homotopy $\mathcal{T}_{n}$-algebra $\underset{\sim}{X}$ with $\underset{\sim}{X}\left(1^{+}\right) \simeq X$.

Definition 5.11 We obtain a morphism of theories $\vartheta_{n}: \mathcal{T}_{n} \rightarrow \mathcal{G}_{1}$ induced by the maps

$$
\mathcal{T}_{n}\left(k^{+}, \ell^{+}\right)=\left(\Omega^{n} \bigvee_{k} S^{n}\right)^{\ell} \rightarrow\left(\operatorname{colim}_{s} \Omega^{n+s} \bigvee_{k} S^{n+s}\right)^{\ell} \simeq \mathcal{G}_{1}\left(k^{+}, \ell^{+}\right)
$$

together with the equation $\vartheta_{n+1} t_{n}=\vartheta_{n}$.

Remark 5.12 The theory $\mathcal{G}_{1}$ is canonically weakly equivalent to the colimit of the sequence

$$
\cdots \longrightarrow \mathcal{T}_{n} \stackrel{t_{n}}{\longrightarrow} \mathcal{T}_{n+1} \stackrel{t_{n+1}}{\longrightarrow} \cdots
$$

in the category of simplicial categories (with fixed set of objects) and hence in the category of simplicial theories.

Theorem 5.13 A pointed space $X$ is an infinite loop space if and only if there exists a homotopy $\mathcal{G}_{1}$-algebra $\underset{\sim}{X}$ with $\underset{\sim}{X}\left(1^{+}\right) \simeq X$. The homotopy category of homotopy 1 -nilpotent groups is equivalent to the homotopy category of infinite loop spaces.

Proof Recall Theorem 5.10. By Theorem 3.5 and Remark 5.12 a space $X$ is an infinite loop space if and only if the associated functor

$$
\underset{\sim}{X}: \Gamma \rightarrow \mathcal{S}_{*}, k^{+} \mapsto X^{k}
$$

extends via the maps $t_{n}$ to a product-preserving functor from $\mathcal{T}_{n}$ for all $n \geq 0$. The existence of the morphism $\vartheta_{n}$ shows that $\underset{\sim}{X}$ is a homotopy $\mathcal{G}_{1}$-algebra if and only if $\underset{\sim}{X}$ restricts to a homotopy $\mathcal{T}_{n}$-algebra for each $n \geq 0$. The equivalence of homotopy categories now also follows.

Definition 5.14 For $1 \leq n \leq \infty$ the maps id $\rightarrow P_{n}$ (id) induce morphisms of theories

$$
\mathcal{G}_{\infty} \rightarrow \mathcal{G}_{n}
$$

that by pulling back along them induce forgetful functors

$$
U_{n}: \mathcal{S}_{*}^{\mathcal{G}_{n}} \rightarrow \mathcal{S}_{*}^{\mathcal{G}_{\infty}} \text {. }
$$

Denote by $R U_{n}$ its right derived functor. These forgetful functors possess left adjoints

$$
\Phi_{n}: \mathcal{S}_{*}^{\mathcal{G}_{\infty}} \rightarrow \mathcal{S}_{*}^{\mathcal{G}_{n}}
$$

We let

$$
L \Phi_{n}: \mathcal{S}_{*}^{\mathcal{G}_{\infty}} \rightarrow \mathcal{S}_{*}^{\mathcal{G}_{n}}
$$


be the enriched homotopy left Kan extension, which is obtained by precomposing $\Phi_{n}$ with a projective cofibrant replacement functor on $\mathcal{S}_{*}^{\mathcal{G}_{\infty}}$. It is the left derived functor of $\Phi_{n}$.

Remark 5.15 (1) One can easily prove that the pair $\left(\Phi_{n}, U_{n}\right)$ forms a Quillen pair for the Badzioch model structures on both sides.

(2) Note that $L \Phi_{n} \underset{\sim}{X}$ depends only on the homotopy $\infty$-nilpotent group structure even when $\underset{\sim}{X}$ has a homotopy $m$-nilpotent group structure for some $m \leq \infty$. For $1 \leq n \leq m \leq \infty$ there exist left adjoint functors

$$
\Phi_{n}^{m}: \mathcal{S}_{*}^{\mathcal{G}_{m}} \rightarrow \mathcal{S}_{*}^{\mathcal{G}_{n}}
$$

that we will consider in future work. It is not true that $L \Phi_{n} R U_{m} \underset{\sim}{X}$ is equivalent to $L \Phi_{n}^{m} \underset{\sim}{X}$.

(3) Note that:

$$
\operatorname{Fr}_{\mathcal{G}_{n}} \cong \Phi_{n} \operatorname{Fr}_{\mathcal{G}_{\infty}}
$$

The object $\operatorname{Fr}_{\mathcal{G}_{\infty}}\left(k^{+}\right) \cong \mathcal{G}_{\infty}\left(k^{+},{ }_{-}\right)$is projectively cofibrant. So Corollary 5.7 says:

$$
L \Phi_{n} \operatorname{Fr}_{\mathcal{G}_{\infty}}\left(k^{+}\right) \simeq \Phi_{n} \operatorname{Fr}_{\mathcal{G}_{\infty}}\left(k^{+}\right) \simeq \Omega P_{n}(\mathrm{id}) \Sigma\left(k^{+}\right) \simeq \Omega P_{n}(\mathrm{id})\left(\bigvee_{k} S^{1}\right)
$$

The next result, Theorem 5.16, can be interpreted as a generalization of this fact.

Theorem 5.16 For any space $K$ in $\mathcal{S}_{*}$ we have a natural weak equivalence

$$
L \Phi_{n} \operatorname{Fr}_{\mathcal{G}_{\infty}} K \simeq \Omega P_{n}(\mathrm{id})(\Sigma K) .
$$

Equivalently, let $\underset{\sim}{X}$ be a homotopy $\mathcal{G}_{\infty}$-algebra with $\underset{\sim}{X}\left(1^{+}\right) \simeq \Omega X$ for some reduced simplicial set $X$. Then there is a natural weak equivalence

$$
\left(L \Phi_{n} \underset{\sim}{X}\right)\left(1^{+}\right) \simeq \Omega P_{n}(\mathrm{id})(X) .
$$

Proof By observing that $\operatorname{Fr}_{\mathcal{G}_{\infty}}(K)=\Omega \Sigma K$ and setting $X=\Sigma K$ the first claim translates into the second one.

In the case $X=\bigvee_{k} S^{1}$ the associated homotopy $\mathcal{G}_{\infty}$-algebra $\underset{\sim}{X}$ is given by

while

$$
\begin{gathered}
\underset{\sim}{X}\left(\ell^{+}\right) \cong\left(\Omega \bigvee_{k} S^{1}\right)^{\ell} \simeq \mathcal{G}_{\infty}\left(k^{+}, \ell^{+}\right), \\
\mathcal{G}_{n}\left(k^{+}, 1^{+}\right) \simeq \Omega P_{n}(\mathrm{id})\left(\bigvee_{k} S^{1}\right)
\end{gathered}
$$


Representable functors are projectively cofibrant and (homotopy) left Kan extension preserves representable functors. So we have an equivalence:

$$
L \Phi_{n} \mathcal{G}_{\infty}\left(k^{+},{ }_{-}\right) \simeq \mathcal{G}_{n}\left(k^{+},{ }_{-}\right)
$$

This proves the case $X=\bigvee_{k} S^{1}$. Now we observe that every reduced finite simplicial set $X$ is weakly equivalent to the realization of a bisimplicial set $X_{\bullet}$, which consists degreewise of a finite wedge of copies of the circle $S^{1}$ : for $k \geq 0$ let $X_{k}$ be the set of $k$-simplices of $X$, and consider $X_{k}$ as a discrete simplicial set. Set $Y_{k}=X_{k+1} \wedge S^{1}$ and recall $X_{0}=*$. Then we have canonical equivalences:

$$
X \simeq\left|k \mapsto X_{k}\right| \simeq\left|k \mapsto Y_{k}\right|
$$

Here $I_{-} \mid$denotes the realization of a bisimplicial set. The statement now follows from Theorem A.2 applied with $F=\Omega P_{n}$ (id) and $\rho=q=1$.

\section{The lower central series of the loop group}

Definition 6.1 Let $G$ be a group. For subgroups $H$ and $K$ of $G$ let $[H, K]$ denote the normal subgroup generated by elements of the form $h^{-1} k^{-1} h k$ where $h \in H$ and $k \in K$. The lower central filtration for $G$ is defined in the following inductive way: Let

$$
\Gamma_{1} G=G \text { and } \Gamma_{n+1} G=\left[G, \Gamma_{n} G\right] .
$$

We obtain a filtration of $G$ by normal subgroups with an associated tower:

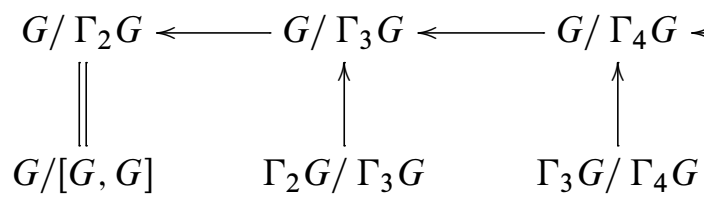

This is the lower central series of $G$. A group $G$ is called $n$-nilpotent if $\Gamma_{n+1} G=0$.

Definition 6.2 For an abelian group $A$ let

$$
\mathrm{Lie}_{*} A=\bigoplus_{n \geq 1} \operatorname{Lie}_{n} A
$$

be the free graded Lie algebra on $A$.

Remark 6.3 The Poincaré-Birkhoff-Witt theorem [32, I.4.3] says that there is a natural isomorphism of abelian groups

$$
\operatorname{Lie}_{n}(G /[G, G]) \cong \Gamma_{n} G / \Gamma_{n+1} G
$$


for every free group $G$. In fact, this group is free abelian on generators given by a Hall basis of basic commutators of weight $n$ over the generators of $G$ [21].

Remember that $\mathcal{S}_{0}$ denotes the category of reduced simplicial sets and $s \mathrm{Gr}$ the category of simplicial groups. Let $G: \mathcal{S}_{0} \rightarrow s \mathrm{Gr}$ be Kan's loop group functor. We can apply the functors from the lower central series degreewise.

Definition 6.4 Let $\Gamma^{n}: \mathcal{S}_{0} \rightarrow \mathcal{S}_{*}$ be the functor given by

$$
\Gamma^{n} X=B\left(G X / \Gamma_{n+1} G X\right) .
$$

The functor $\bar{\Gamma}^{n}: \mathcal{S}_{0} \rightarrow \mathcal{S}_{*}$ will be given by

$$
\bar{\Gamma}^{n} X=B\left(\Gamma_{n} G X / \Gamma_{n+1} G X\right) .
$$

Remark 6.5 The loop group is a free simplicial group. It follows from a theorem by Dold [13] that both functors $\Gamma^{n}$ and $\bar{\Gamma}^{n}$ preserve weak equivalences. Moreover with Remark 6.3 we have a formula:

$$
\bar{\Gamma}^{n} X \simeq B \operatorname{Lie}_{n}(\widetilde{\mathbb{Z}} X)
$$

Here $\widetilde{\mathbb{Z}} X=\mathbb{Z} X / \mathbb{Z} *$ is the reduced free simplicial abelian group on $X$.

Remark 6.6 It is proved by Curtis [12] that for a simply connected space $X$ the map

$$
X \simeq B G X \rightarrow B\left(G X / \Gamma_{n} G X\right)=\Gamma^{n-1} X
$$

is $\left\{\log _{2} n\right\}$-connected where $\{a\}$ is the least integer $\geq a$. If $X$ is merely connected, the tower $\left\{\Gamma^{n}(X)\right\}_{n \in \mathbb{N}}$ converges to the Bousfield-Kan completion $\mathbb{Z}_{\infty}(X)$. Compare Remark 7.1 about the Goodwillie tower of the identity.

For $n=1$ we have $\bar{\Gamma}^{1} X=B \widetilde{\mathbb{Z}} X$. This functor is linear, because we have for all $s \geq 0$ :

$$
\pi_{s} B \widetilde{\mathbb{Z}} X \cong \tilde{H}_{S} X
$$

Here $\widetilde{H}_{*} X$ is the reduced singular homology of the reduced space $X$. More generally, there is the following lemma.

Lemma 6.7 The functor $\bar{\Gamma}^{n}$ is $n$-excisive.

Proof Consider for free abelian groups $A_{1}, \ldots, A_{n+1}$ the cubical diagram

$$
P(\underline{n+1}) \rightarrow \text { FrAb }, \quad S \mapsto \bigoplus_{i \in \underline{n+1}-S} A_{i},
$$


where the maps are induced by collapsing summands. The $(n+1)-$ st cross effect of the functor $\mathrm{Lie}_{n}: \mathrm{FrAb} \rightarrow \mathrm{Ab}$ vanishes because the associated complex

$$
k \mapsto \bigoplus_{|S|=k} \operatorname{Lie}_{n}\left(\bigoplus_{i \in \underline{n+1}-S} A_{i}\right)=: L_{k}
$$

is exact. It follows that the composition

$$
\bar{\Gamma}^{n} X=B \operatorname{Lie}_{n}(\widetilde{\mathbb{Z}} X)
$$

is $n$-excisive.

Remark 6.8 It is not true though that the functor $\bar{\Gamma}^{n}$ is $n$-homogeneous. By Curtis' result Remark 6.6 the tower $\left\{\Gamma^{n}\right\}_{n \geq 0}$ converges to the identity on simply connected spaces. This shows that the layers of the tower have to contribute something to the linear part given by reduced homology $\widetilde{H}_{*}$ in order to make it up to the first derivative of the identity given by stable homotopy $\pi_{*}^{\text {st }}$.

Corollary 6.9 The functor $\Gamma^{n}$ is $n$-excisive.

Proof There is a homotopy fiber sequence

$$
\Gamma^{n} \rightarrow \Gamma^{n-1} \rightarrow B \bar{\Gamma}^{n}
$$

of functors. By induction the statement follows from Lemma 6.7 .

\section{The Goodwillie tower of the identity}

We need to gather some remarks on the Goodwillie tower of the identity.

Remark 7.1 The identity functor is 1-analytic [19], which shows that its Goodwillie tower converges on simply connected spaces to the identity. However, on connected spaces $X$ it converges to the Bousfield-Kan completion of $X$ :

$$
\underset{n}{\operatorname{holim}} P_{n}(\mathrm{id})(X) \simeq \mathbb{Z}_{\infty} X
$$

This is proved on the last page of Arone and Kankaanrinta [3].

Definition 7.2 Let $D_{n}$ (id) $\left(\bigvee_{k} S^{1}\right)$ be the homotopy fiber of the map

$$
P_{n}(\mathrm{id})\left(\bigvee_{k} S^{1}\right) \rightarrow P_{n-1}(\mathrm{id})\left(\bigvee_{k} S^{1}\right)
$$


in the Goodwillie tower. Then $D_{n}(\mathrm{id})\left(\bigvee_{k} S^{1}\right)=\Omega^{\infty} E_{n}$ for the following spectrum

$$
\left(\partial^{n}(\mathrm{id}) \wedge\left(\bigvee_{k} S^{1}\right)^{\wedge n}\right)_{h \Sigma_{n}}=: E_{n},
$$

where $\partial^{n}$ (id) is the $n$-th derivative of the identity.

Lemma 7.3 The rational homology of $E_{n}$ is concentrated in degree 1 .

Proof There is a Serre spectral sequence:

$$
H_{i}\left(\Sigma_{n}, H \mathbb{Q}_{j}\left(\partial^{n}(\mathrm{id}) \wedge\left(\bigvee_{k} S^{1}\right)^{\wedge n}\right)\right) \Longrightarrow H \mathbb{Q}_{i+j}\left(E_{n}\right)
$$

The homology of $\Sigma_{n}$ with coefficients in a rational vector space vanishes for $i>0$ and gives the formula:

$$
H \mathbb{Q}_{*} E_{n} \cong H \mathbb{Q}_{*}\left(\partial^{n}(\mathrm{id})\right) \otimes_{\mathbb{Q}\left[\Sigma_{n}\right]} H \mathbb{Q}_{*}\left(\left(\bigvee_{k} S^{1}\right)^{\wedge n}\right) .
$$

The homology of $\left(\bigvee_{k} S^{1}\right)^{\wedge n}$ is concentrated in degree $n$. By the work of Johnson [24] and Arone and Mahowald [4] we know that the spectrum $\partial^{n}$ (id) is nonequivariantly equivalent to $\bigvee_{(n-1) !} S^{1-n}$ and so has homology concentrated in degree $1-n$. So $1-n+n=1$, and the statement follows.

Lemma 7.4 The group $\pi_{1} P_{n}(\mathrm{id})\left(\bigvee_{k} S^{1}\right)$ is nilpotent of degree $n$.

Proof We can settle the case $n=1$ right away:

$$
\pi_{0} \Omega P_{1}(\mathrm{id})\left(\bigvee_{k} S^{1}\right) \cong \pi_{1}\left(\Omega^{\infty} \Sigma^{\infty} \bigvee_{k} S^{1}\right) \cong \mathbb{Z}^{k} \cong F_{k} / \Gamma_{2} F_{k}
$$

In particular, this group is nilpotent of degree 1 . We proceed by induction on $n$. From the Goodwillie tower we have for each $n \geq 1$ the following exact sequence of groups:

$$
\begin{aligned}
\pi_{1} D_{n}(\mathrm{id})\left(\bigvee_{k} S^{1}\right) & \rightarrow \pi_{1} P_{n}(\mathrm{id})\left(\bigvee_{k} S^{1}\right) \rightarrow \pi_{1} P_{n-1}(\mathrm{id})\left(\bigvee_{k} S^{1}\right) \\
& \rightarrow \pi_{0} D_{n}(\mathrm{id})\left(\bigvee_{k} S^{1}\right) \cong 0
\end{aligned}
$$


The last group vanishes because of Lemma 7.3. By Goodwillie's results [20] the spaces $D_{n}(\mathrm{id})\left(\bigvee_{k} S^{1}\right)$ are infinite loop spaces and the map in the Goodwillie tower is a principal fibration, ie there is a homotopy pullback square

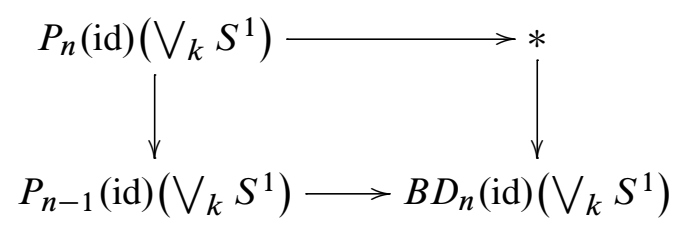

where $B D_{n}(\mathrm{id})\left(\bigvee_{k} S^{1}\right)$ is a delooping of $D_{n}(\mathrm{id})\left(\bigvee_{k} S^{1}\right)$ and therefore simply connected. Let

$$
K_{n}=\operatorname{im}\left(\pi_{1} D_{n}(\mathrm{id})\left(\bigvee_{k} S^{1}\right) \rightarrow \pi_{1} P_{n}(\mathrm{id})\left(\bigvee_{k} S^{1}\right)\right)
$$

Then the short exact sequence

$$
0 \rightarrow K_{n} \rightarrow \pi_{1} P_{n}(\mathrm{id})\left(\bigvee_{k} S^{1}\right) \rightarrow \pi_{1} P_{n-1}(\mathrm{id})\left(\bigvee_{k} S^{1}\right) \rightarrow 0
$$

is a central extension. It follows inductively that the group $\pi_{1} P_{n}(\mathrm{id})\left(\bigvee_{k} S^{1}\right)$ is nilpotent of degree $n$.

Lemma 7.5 The groups $\pi_{s} P_{n}(\mathrm{id})\left(\bigvee_{k} S^{1}\right)$ are finite for $s \geq 2$.

Proof These groups are finitely generated. So it is enough to prove that the groups $\pi_{s} P_{n}(\mathrm{id})\left(\bigvee_{k} S^{1}\right)$ are torsion above degree $s=1$. We will prove this by induction along the Goodwillie tower where the case $n=0$ is obvious, because the space is contractible. Next we know by Lemma 7.3 that the rational homology of $D_{n}(\mathrm{id})\left(\bigvee_{k} S^{1}\right)$ is concentrated in degree 1 . Since $D_{n}(\mathrm{id})\left(\bigvee_{k} S^{1}\right)$ is an infinite loop space, a form of the Hurewicz theorem [33, Theorem 9.6.20] tells us that also the groups $\pi_{s} D_{n}(\mathrm{id})\left(\bigvee_{k} S^{1}\right)$ are torsion for $s>1$. The result now follows from the long exact homotopy sequence of the Goodwillie tower.

Corollary 7.6 There is an isomorphism of groups:

$$
\pi_{1} \operatorname{holim}_{n} P_{n}(\mathrm{id})\left(\bigvee_{k} S^{1}\right) \cong \lim _{n} \pi_{1} P_{n}(\mathrm{id})\left(\bigvee_{k} S^{1}\right)
$$


Proof There is the Milnor exact sequence:

$$
\begin{aligned}
0 \rightarrow \lim _{n}^{1} \pi_{2} P_{n}(\mathrm{id})\left(\bigvee_{k} S^{1}\right) \rightarrow \pi_{1} \operatorname{holim}_{n} P_{n}(\mathrm{id}) & \left(\bigvee_{k} S^{1}\right) \\
& \rightarrow \lim _{n} \pi_{1} P_{n}(\mathrm{id})\left(\bigvee_{k} S^{1}\right) \rightarrow 0
\end{aligned}
$$

By Lemma 7.5 the $\lim ^{1}$-term vanishes.

The following conjecture is related to the vanishing of $\lim ^{1} \pi_{2} P_{n}$ (id) $\left(\bigvee_{k} S^{1}\right)$.

Conjecture 7.7 (Arone-Mahowald-Kuhn) For each prime $p$ the map

$$
\pi_{S} P_{p^{n}}(\mathrm{id})\left(S^{1}\right)_{(p)} \rightarrow \pi_{S} P_{p^{n-1}}(\mathrm{id})\left(S^{1}\right)_{(p)}
$$

is null for $s \geq 2$.

\section{Relation to ordinary nilpotent groups}

Now we can describe the relation of $\mathcal{G}_{n}$ to the set-valued theory of ordinary $n$-nilpotent groups $\mathrm{Nil}_{n}$, whose $k$-ary operations are given by the free $n$-nilpotent group on $k$ generators:

$$
\operatorname{Nil}_{n}\left(k^{+}, 1^{+}\right)=F_{k} / \Gamma_{n+1} F_{k}
$$

Here $F_{k}$ is the free group on $k$ generators. We can exhibit this theory by applying $\pi_{0}$ to the theory of homotopy $n$-nilpotent groups. First observe that for the case $n=\infty$ the statement

$$
\pi_{0} \mathcal{G}_{\infty}\left(k^{+}, 1^{+}\right)=\pi_{1}\left(\bigvee_{k} S^{1}\right) \cong F_{k} \cong \operatorname{Nil}_{\infty}\left(k^{+}, 1^{+}\right)
$$

follows from the Seifert-Van Kampen theorem. This isomorphism has an analogue for finite $n$. There is a map

$$
F_{k} \cong \pi_{1}\left(\bigvee_{k} S^{1}\right) \rightarrow \pi_{1} P_{n}(\mathrm{id})\left(\bigvee_{k} S^{1}\right) \cong \pi_{0} \mathcal{G}_{n}\left(k^{+}, 1^{+}\right)
$$

induced by the natural transformation id $\rightarrow P_{n}$ (id), which factors through

$$
\alpha_{n}: F_{k} / \Gamma_{n+1} F_{k} \rightarrow \pi_{1} P_{n}(\mathrm{id})\left(\bigvee_{k} S^{1}\right),
$$

because the target is $n$-nilpotent by Lemma 7.4. 
Theorem 8.1 We have an isomorphism of groups:

$$
\alpha_{n}: \operatorname{Nil}_{n}\left(k^{+}, 1^{+}\right)=F_{k} / \Gamma_{n+1} F_{k} \cong \pi_{1} P_{n}(\mathrm{id})\left(\bigvee_{k} S^{1}\right) \cong \pi_{0} \mathcal{G}_{n}\left(k^{+}, 1^{+}\right)
$$

This induces an isomorphism of categories $\mathrm{Nil}_{n} \cong \pi_{0} \mathcal{G}_{n}$.

Proof We show first that $\alpha_{n}$ is injective by constructing a left inverse $\beta_{n}$. According to Corollary 6.9 the functor $\Gamma^{n}$ is $n$-excisive. So there is a natural transformation $P_{n}($ id $) \rightarrow \Gamma^{n}$ under the identity functor. If we evaluate this diagram on $\bigvee_{k} S^{1}$ and apply $\pi_{1} \cong \pi_{0} G$, we obtain a map $b_{n}$ making the following diagram commutative:

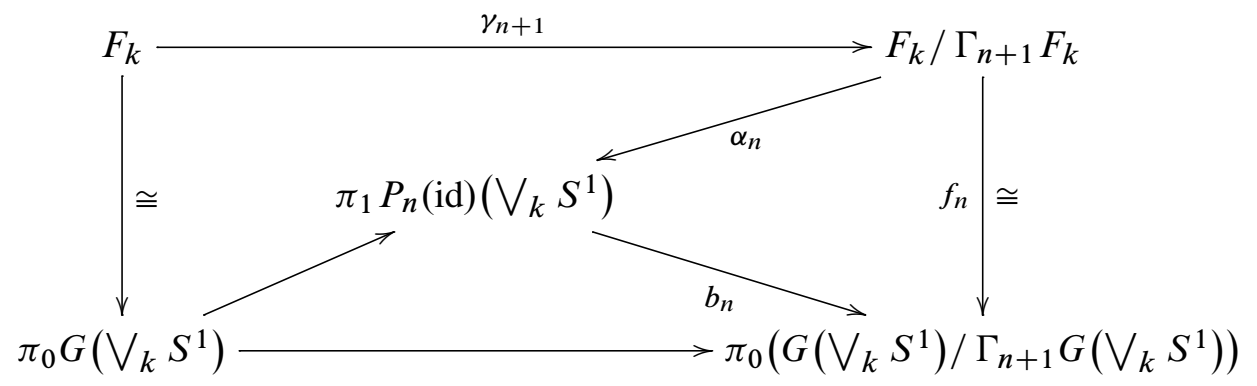

Let $\beta_{n}=f_{n}^{-1} b_{n}$. It follows that $\beta_{n} \alpha_{n} \gamma_{n+1}=\gamma_{n+1}$. Since $\gamma_{n+1}$ is the universal map into an $n$-nilpotent group, we have $\beta_{n} \alpha_{n}=\mathrm{id}$.

But the map $\alpha_{n}$ is also surjective. Let $Q_{n}$ be the quotient of $\alpha_{n}$, ie the pointed set of left cosets. We obtain a short exact sequence of towers:

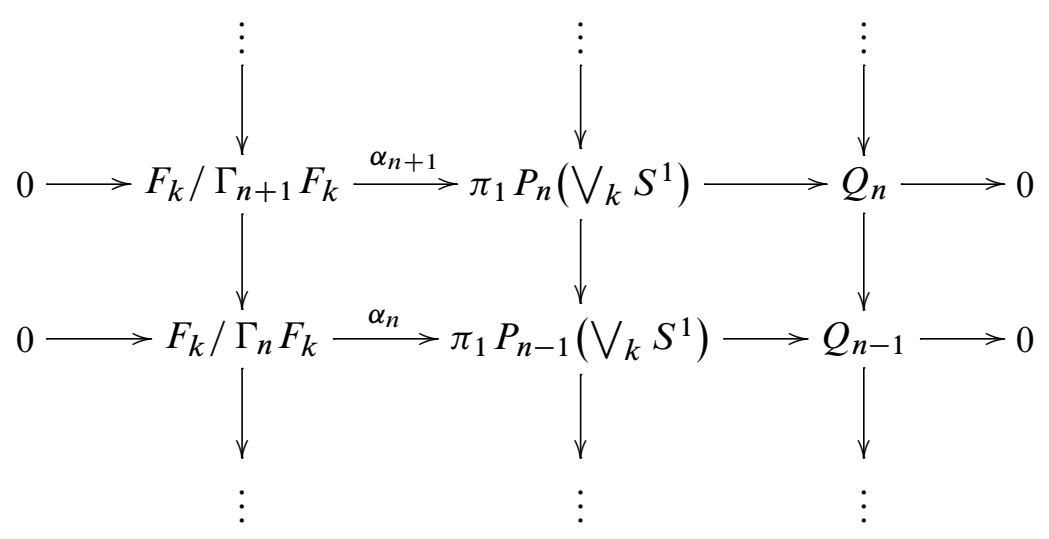


From (3) it follows that the vertical maps are surjective for $n \geq 1$. So all $\lim ^{1}$-terms vanish. In the limit we obtain a short exact sequence:

$$
0 \rightarrow \lim _{n} F_{k} / \Gamma_{n+1} F_{k} \rightarrow \lim _{n} \pi_{1} P_{n}(\mathrm{id})\left(\bigvee_{k} S^{1}\right) \rightarrow \lim _{n} Q_{n} \rightarrow 0
$$

We can combine the weak equivalence (2) with the isomorphism from Corollary 7.6 to conclude that $\lim _{n} Q_{n} \cong *$. In turn we have $Q_{n}=*$ for all $n \geq 1$, since all tower maps are surjective. So each $\alpha_{n}$ is an isomorphism.

\section{Values of $n$-excisive functors}

We will prove that functors of the form $\Omega F$ with $F n$-excisive naturally take values in the category of homotopy $n$-nilpotent groups. We take this as a justification of the usefulness of the notion of homotopy $n$-nilpotent groups.

We need to compose functors. But two functors $F$ and $G$ in $\mathcal{F}$ cannot be composed directly. However, we can extend the functor $F: \mathcal{S}_{*}^{\text {fin }} \rightarrow \mathcal{S}_{*}$ to a functor $\mathcal{S}_{*} \rightarrow \mathcal{S}_{*}$ by enriched left Kan extension. By abuse of language we will denote this functor again by $F$. Then the composition $F \circ G$ is well-defined.

Observe that the functor $\_\circ G: \mathcal{F} \rightarrow \mathcal{F}$ commutes with finite limits. And there is a functor

$$
P_{n}\left(\_\circ G\right): \mathcal{F} \rightarrow \mathcal{F}, F \mapsto P_{n}(F \circ G),
$$

which also commutes with finite limits. The map $F \rightarrow P_{n} F$ induces a map

$$
P_{n}(F \circ G) \rightarrow P_{n}\left(\left(P_{n} F\right) \circ G\right)
$$

under $F \circ G$.

Lemma 9.1 The map (5) is an objectwise weak equivalence.

This is proved directly by Michael Ching in [10, Proposition 6.1(1)]. It is also a consequence of the chain rule (6) which is now a theorem by Arone and Ching [1].

For the next theorem we point out that the language of simplicial algebraic theories and (homotopy) algebras over them makes sense in any cartesian closed symmetric monoidal $\mathcal{S}_{*}$-model category. The category $\mathcal{F}$ of $\mathcal{S}_{*}$-functors from $\mathcal{S}_{*}^{\text {fin }}$ to $\mathcal{S}_{*}$ equipped with the categorical product as the monoidal structure is such an example.

Theorem 9.2 Let $F$ be a functor of the form $F=\Omega G$ with an $n$-excisive functor $G$ in $\mathcal{F}$. Then $F$ is a homotopy $\mathcal{G}_{n}$-algebra in $\mathcal{F}$. 
Proof A homotopy $\mathcal{G}_{n}$-algebra in $\mathcal{F}$ is an $\mathcal{S}_{*}$-functor $\mathcal{G}_{n} \rightarrow \mathcal{F}$ preserving products up to weak equivalence. Given $F$ as above we define the object function of a functor $\underset{\sim}{F}: \mathcal{G}_{n} \rightarrow \mathcal{F}$ by

$$
k^{+} \mapsto F^{k}=\underset{\sim}{F}\left(k^{+}\right) .
$$

To see that this actually yields an $\mathcal{S}_{*}$-enriched functor we observe that this is the object function of the following composition of functors: First we take the full inclusion functor $I_{n}: \mathcal{G}_{n} \rightarrow \mathcal{F}$

$$
k^{+} \mapsto I_{n}\left(k^{+}\right)=\prod_{k} \Omega P_{n}(\mathrm{id}) .
$$

Then we precompose with $G$ :

$$
\prod_{k} \Omega P_{n}(\mathrm{id}) \mapsto \prod_{k} \Omega P_{n}(\mathrm{id}) \circ G
$$

Finally we apply $P_{n}: \mathcal{F} \rightarrow \mathcal{F}$ :

$$
\prod_{k} \Omega P_{n}(\mathrm{id}) \circ G \mapsto P_{n}\left(\left(\prod_{k} \Omega P_{n}(\mathrm{id})\right) \circ G\right)
$$

Note that the inclusion $I_{n}$ as well as the functors $\_\circ G$ and $P_{n}$ commute with products up to weak equivalence, and the latter two functors commute both up to homotopy with $\Omega$. We compute using Lemma 9.1:

$$
P_{n}\left(\left(\prod_{k} \Omega P_{n}(\mathrm{id})\right) \circ G\right) \simeq \prod_{k} \Omega P_{n}\left(P_{n}(\mathrm{id}) \circ G\right) \simeq \prod_{k} \Omega P_{n}(G) \simeq F^{k}
$$

This shows at the same time that the object function of $\underset{\sim}{F}$ is part of an $\mathcal{S}_{*}$-functor $\mathcal{G}_{n} \rightarrow \mathcal{F}$ and that this functor preserves products up to weak equivalence. Hence $\underset{\sim}{F}$ is indeed a homotopy $\mathcal{G}_{n}$-algebra in $\mathcal{F}$.

Corollary 9.3 Let $F$ be a functor of the form $F=\Omega G$ with an $n$-excisive functor $G$ in $\mathcal{F}$. Then for any $K$ in $\mathcal{S}_{*}^{\text {fin }}$ the space $F(K)$ is a homotopy $n$-nilpotent group.

Proof For any space $K$ the evaluation functor $\operatorname{Ev}_{K}: \mathcal{F} \rightarrow \mathcal{S}_{*}$ preserves products. So the statement is a corollary of Theorem 9.2.

Work in progress [8] by the two authors shows that the converse of Corollary 9.3 is true: one can associate to any homotopy $n$-nilpotent group $\underset{\sim}{X}$ an $n$-excisive functor $F$ such that $F\left(S^{0}\right) \simeq \mathrm{Ev}_{1}+\underset{\sim}{X}$.

The following statement is also a formal consequence of Theorem 9.2. 
Corollary 9.4 Let $G$ be a functor of the form $G=\Omega H$ with an $n$-excisive functor $H$ in $\mathcal{F}$. Let $F$ be an arbitrary functor in $\mathcal{F}$. Then $\mathcal{F}(F, G)$ is a homotopy $n$-nilpotent group naturally in $F$ and $G$.

Corollary 9.4 says that the full subcategory of $\mathcal{F}$ of functors of the form $\Omega F$ with $F$ being $n$-excisive is enriched over homotopy $n$-nilpotent groups. In particular, by Theorem 8.1 we obtain the following statement.

Corollary 9.5 The full subcategory of the homotopy category of $\mathcal{F}$ given by functors of the form $\Omega F$ with $F n$-excisive is enriched over $n$-nilpotent groups.

This generalizes the fact that in the case $n=1$ the stable homotopy category is enriched over abelian groups.

We would like to finish with two more examples. In work in progress [8] we are going to study closer classifying spaces of homotopy $n$-nilpotent groups. This point of view is more convenient in the following examples.

Definition 9.6 A space is called a homotopy n-nilpotent space if it is given as the delooping or classifying space of a homotopy $n$-nilpotent group.

Remark 9.7 One can easily see that a homotopy $n$-nilpotent space is exactly a homotopy algebra over the simplicial theory $\mathcal{P}_{n}$ given by

$$
\mathcal{P}_{n}\left(k^{+}\right):=\prod_{k} P_{n}(\mathrm{id})^{\mathrm{inj}} \subset \mathcal{F} .
$$

A proof will appear in [8].

Example 9.8 Let $X$ be a pointed connected space whose homotopy groups $\pi_{s} X$ vanish unless $k+1 \leq s \leq(n+1) k$ for some $n, k \geq 1$. In the case $n=1$ it follows from the classical Blakers-Massey theorem that $X \simeq$ Post $_{2 k} \Omega^{\infty} \Sigma^{\infty} X$. Here Post ${ }_{s} X$ denotes the $s$-th Postnikov section of $X$ which preserves infinite loop spaces. Hence $X$ is an infinite loop spaces. For general $n$ it follows from the generalized Blakers-Massey theorem due to Ellis and Steiner [17] or Goodwillie [19, Theorem 2.3], that

$$
X \simeq \operatorname{Post}_{(n+1) k} P_{n}(\mathrm{id})(X) .
$$

By Corollary 9.3 the space $P_{n}$ (id) $X$ has a structure as homotopy $n$-nilpotent space. Postnikov sections of homotopy $n$-nilpotent groups remain homotopy $n$-nilpotent groups. This can be proved eg by observing that the Moore model for Postnikov sections [18, VI.3.4.] commutes with products on the nose. It follows, that $X$ can be given the structure of a homotopy $n$-nilpotent space. Its loop space $\Omega X$ is canonically a homotopy $n$-nilpotent group. 
For the next example we rely on the chain rule in Goodwillie's calculus of homotopy functors. Let $\partial^{j} F$ denote the $j$-th derivative of $F$ at $*$ and note that all the derivatives $\partial^{*} F$ together form a symmetric sequence in spectra. It is known by Ching [11] that the derivatives of the identity $\partial^{*}$ id form an operad. In [1] Arone and Ching prove that the derivatives of any homotopy functor $F$ in $\mathcal{F}$ form a left and right module over $\partial^{*}$ id and that the canonical map

$$
\partial^{*}(G \circ F) \simeq \partial^{*} G \circ \partial^{*} \text { id } \partial^{*} F
$$

is a weak equivalence. The circle product on the right side is induced by the composition product of symmetric sequences. For a different approach in the spectrum-valued case we refer to Ching [10]. A simple application is the following statement.

Corollary 9.9 Let $n, k \geq 1$. Let $G \rightarrow H$ be an $n$-excisive equivalence in $\mathcal{F}$ and $F$ be a functor with $\partial^{j} F \simeq *$ for $j \leq k$. Then the induced map

$$
G \circ F \rightarrow H \circ F
$$

is an $(n k+n+k)$-excisive equivalence.

Example 9.10 Let $F$ be a functor in $\mathcal{F}$ with $\partial^{j} F \simeq *$ unless $k+1 \leq j \leq n k+n+k$ for some $n, k \geq 1$. Then $F$ factors through the category of homotopy $n$-nilpotent spaces.

As in the proof of Theorem 9.2 we will use homotopy $\mathcal{P}_{n}$-algebras in $\mathcal{F}$. We note that the $s$-th Goodwillie section $P_{s} L$ of a functor $L$ in $\mathcal{F}$ remains a homotopy $\mathcal{P}_{n}$-algebra if $L$ itself was one, because $P_{S}$ commutes with finite homotopy limits in $\mathcal{F}$ by [20, 1.7.]. The map id $\rightarrow P_{n}$ (id) is an $n$-excisive equivalence. Precomposing with $F$ yields an equivalence

$$
F \rightarrow P_{n k+n+k} F \rightarrow P_{n k+n+k}\left(P_{n}(\mathrm{id}) \circ F\right)
$$

by Corollary 9.9. By the proof of Corollary 9.3 the functor $P_{n}$ (id) $\circ F$ is a homotopy $\mathcal{P}_{n}$-algebra, and so is $F$. In particular $F$ takes values in homotopy $n$-nilpotent spaces. By looping the setup we return to homotopy $n$-nilpotent groups.

The case $n=1$ of the previous example was proved in [2, Theorem 4.2].

\section{Appendix A An auxiliary lemma about realizations}

Let us quickly review some notions introduced by Goodwillie in [19, Definition 4.1]. 
Definition A.1 A functor $F$ in $\mathcal{F}$ is said to satisfy condition $E_{n}(c, \kappa)$ if there exist constants $c$ and $\kappa$ both in $\mathbb{Z}$ and $\kappa \geq-1$ such that for every strongly homotopy cocartesian $(n+1)$-cube $\mathcal{C}$ whose maps $\mathcal{C}(\varnothing) \rightarrow \mathcal{C}(\{s\})$ are $k_{s}$-connected with $k_{s} \geq \kappa$ the induced map

$$
F(\mathcal{C}(\varnothing)) \rightarrow \operatorname{holim}_{S \in P_{0}(\underline{n+1})} F(\mathcal{C}(S))
$$

is $\left(-c+\Sigma k_{s}\right)$-connected. A functor is stably $n$-excisive if it satisfies $E_{n}(c, \kappa)$ for some $c$ and $\kappa$. A functor is $\rho$-analytic if there exists a constant $q \in \mathbb{Z}$ such that $F$ satisfies condition $E_{n}(n \rho-q, \rho+1)$ for all $n \geq 1$.

The first author learned the next statement from explanations of the second author and from the unpublished version [28] of Andrew Mauer-Oats' thesis. The published version is [29].

Theorem A.2 Let $F$ be a reduced finitary $\rho$-analytic functor from spaces to spaces. If $X \bullet$ is a simplicial object in $k$-connected spaces with $k \geq \max (\rho,-q)$ then the canonical map

$$
\left|F X_{\bullet}\right| \stackrel{\simeq}{\rightarrow} F\left|X_{\bullet}\right|
$$

is a weak equivalence.

A sketch of the proof goes as follows: First one observes that homogeneous functors with connective coefficient spectrum commute with realizations. Then the theorem follows by induction up the Goodwillie tower. All along one checks that the connectivity estimates allow one to apply theorem [9, B.4] that gives sufficient conditions for the realization functor to commute with pullbacks.

\section{References}

[1] G Arone, M Ching, Operads and chain rules for the calculus of functors arXiv: 0902.0399

[2] G Arone, W G Dwyer, K Lesh, Loop structures in Taylor towers, Algebr. Geom. Topol. 8 (2008) 173-210 MR2377281

[3] G Arone, M Kankaanrinta, A functorial model for iterated Snaith splitting with applications to calculus of functors, from: "Stable and unstable homotopy (Toronto, ON, 1996)", (W G Dwyer, S Halperin, R Kane, S O Kochman, ME Mahowald, P S Selick, editors), Fields Inst. Commun. 19, Amer. Math. Soc. (1998) 1-30 MR1622334

[4] G Arone, M Mahowald, The Goodwillie tower of the identity functor and the unstable periodic homotopy of spheres, Invent. Math. 135 (1999) 743-788 MR1669268 
[5] B Badzioch, Algebraic theories in homotopy theory, Ann. of Math. (2) 155 (2002) 895-913 MR1923968

[6] B Badzioch, K Chung, A A Voronov, The canonical delooping machine, J. Pure Appl. Algebra 208 (2007) 531-540 MR2277692

[7] G Biedermann, B Chorny, O Röndigs, Calculus of functors and model categories, Adv. Math. 214 (2007) 92-115 MR2348024

[8] G Biedermann, W G Dwyer, Homotopy nilpotent spaces, work in progress

[9] A K Bousfield, E M Friedlander, Homotopy theory of $\Gamma$-spaces, spectra, and bisimplicial sets, from: "Geometric applications of homotopy theory (Proc. Conf., Evanston, Ill., 1977), II”, Lecture Notes in Math. 658, Springer, Berlin (1978) 80-130 MR513569

[10] M Ching, A chain rule for Goodwillie derivatives of functors from spectra to spectra, to appear in Trans. Amer. Math. Soc. arXiv:math. AT/07105567

[11] M Ching, Bar constructions for topological operads and the Goodwillie derivatives of the identity, Geom. Topol. 9 (2005) 833-933 MR2140994

[12] E B Curtis, Some relations between homotopy and homology, Ann. of Math. (2) 82 (1965) 386-413 MR0184231

[13] A Dold, Homology of symmetric products and other functors of complexes, Ann. of Math. (2) 68 (1958) 54-80 MR0097057

[14] W G Dwyer, Localizations, from: "Axiomatic, enriched and motivic homotopy theory", (J P C Greenlees, editor), NATO Sci. Ser. II Math. Phys. Chem. 131, Kluwer Acad. Publ., Dordrecht (2004) 3-28 MR2061850

[15] W G Dwyer, D M Kan, Homotopy theory and simplicial groupoids, Nederl. Akad. Wetensch. Indag. Math. 46 (1984) 379-385 MR770723

[16] W G Dwyer, D M Kan, Equivalences between homotopy theories of diagrams, from: "Algebraic topology and algebraic $K$-theory (Princeton, N.J., 1983)", (W Browder, editor), Ann. of Math. Stud. 113, Princeton Univ. Press (1987) 180-205 MR921478

[17] G Ellis, R Steiner, Higher-dimensional crossed modules and the homotopy groups of $(n+1)-a d s$, J. Pure Appl. Algebra 46 (1987) 117-136 MR897011

[18] PG Goerss, J F Jardine, Simplicial homotopy theory, Progress in Math. 174, Birkhäuser Verlag, Basel (1999) MR1711612

[19] T G Goodwillie, Calculus. II. Analytic functors, K-Theory 5 (1992) 295-332 MR1162445

[20] T G Goodwillie, Calculus. III. Taylor series, Geom. Topol. 7 (2003) 645-711 MR2026544

[21] M Hall, Jr, A basis for free Lie rings and higher commutators in free groups, Proc. Amer. Math. Soc. 1 (1950) 575-581 MR0038336 
[22] PS Hirschhorn, Model categories and their localizations, Math. Surveys and Monogr. 99, Amer. Math. Soc. (2003) MR1944041

[23] J F Jardine, Simplicial presheaves, J. Pure Appl. Algebra 47 (1987) 35-87 MR906403

[24] B Johnson, The derivatives of homotopy theory, Trans. Amer. Math. Soc. 347 (1995) 1295-1321 MR1297532

[25] A Joyal, Letter to A Grothendieck

[26] N J Kuhn, Goodwillie towers and chromatic homotopy: an overview, from: "Proceedings of the Nishida Fest (Kinosaki 2003)", (M Ando, N Minami, J Morava, W S Wilson, editors), Geom. Topol. Monogr. 10 (2007) 245-279 MR2402789

[27] F W Lawvere, Functorial semantics of algebraic theories, Proc. Nat. Acad. Sci. U.S.A. 50 (1963) 869-872 MR0158921

[28] A Mauer-Oats, Goodwillie calculi, $\mathrm{PhD}$ thesis, University of Illinois UrbanaChampaign (2002)

[29] A Mauer-Oats, Algebraic Goodwillie calculus and a cotriple model for the remainder, Trans. Amer. Math. Soc. 358 (2006) 1869-1895 MR2197433

[30] C Reedy, Homology of algebraic theories, PhD thesis, University of California, San Diego (1974)

[31] S Schwede, Stable homotopy of algebraic theories, Topology 40 (2001) 1-41 MR1791267

[32] J-P Serre, Lie algebras and Lie groups, second edition, Lecture Notes in Math. 1500, Springer (1992) MR1176100 1964 lectures given at Harvard University

[33] E H Spanier, Algebraic topology, McGraw-Hill, New York (1966) MR0210112

Departamento de Matemáticas, Universidad de los Andes

Cr 1a No 18A 10, Bogotá, Colombia

Department of Mathematics, 255 Hurley, University of Notre Dame

Notre Dame IN 46556, USA

georgbiedermann@yahoo.com, gbiederm@uniandes.edu.co, dwyer.1@nd.edu http://matematicas.uniandes.edu.co/ gbiederm, http://www. math.nd.edu/ dwyer

Received: 16 September 2009 Revised: 2 October 2009 